\title{
Enhanced Pyroelectric coefficient of Antiferroelectric-Ferroelectric Bi-layer thin films
}

\author{
S. Corkovic and Q. Zhang \\ Department of Materials, Cranfield University, Bedfordshire, MK43 OAL
}

United Kingdom

\begin{abstract}
In this study, the pyroelectric coefficient and the figure of merit (FOM) of the ferroelectric $\mathrm{PbZr}_{0.3} \mathrm{Ti}_{0.7} \mathrm{O}_{3}$ (PZT 30/70) thin films were found to be greatly enhanced by introducing a thin antiferroelectric $\mathrm{PbZr}_{0.95} \mathrm{Ti}_{0.05} \mathrm{O}_{3}$ (PZT 95/05) layer underneath the ferroelectric film and thus creating a bi-layer structure on platinised silicon substrates. The film properties were investigated as a function of the ferroelectric layer thickness when the thickness of antiferroelectric layer remained unchanged. The highest pyroelectric coefficient of $1 \mu \mathrm{m}$ thick PZT 30/70 film was $3.18 \times 10^{-4} \mathrm{Cm}^{-2} \mathrm{~K}^{-1}$. However, the highest pyroelectric coefficient for $1 \mu \mathrm{m}$ thick bi-layer film was $3.5 \times 10^{-4} \mathrm{Cm}^{-2} \mathrm{~K}^{-1}$ or $2.5 \times 10^{-4} \mathrm{Cm}^{-2} \mathrm{~K}^{-1}$ for only $280 \mathrm{~nm}$ thick bi-layer film. The enhancement of pyroelectric coefficient suggests switching of antiferroelectric (AF) into ferroelectric (FE) phase during poling and following stabilization of FE phase at room temperature. The reduction of dielectric constant in bi-layer films after poling, compared to pure PZT 30/70, showed a FOM of $2.94 \times 10^{-5} \mathrm{~Pa}^{-0.5}$ which is the double of the FOM for pure PZT $30 / 70$ films of similar thickness $\left(1.45 \times 10^{-5} \mathrm{~Pa}^{-0.5}\right)$.
\end{abstract}

\section{Introduction}

Recently the antiferroelectric PZT films (AF) have attracted some attention as films for high strain microelectromechanical systems application [1-2] or high charge storage devices due to its 
field induced antiferroelectric-to-ferroelectric phase transition accompanied with high strain [35]. Heterolayered films by alternating coatings of two types of materials of similar composition but different electrical properties has showed promising results such as improved ferroelectric fatigue or reduced dielectric constant compared to homogeneous films of one composition [6-8]. Furthermore, heterolayered rhombohedral and tetragonal films showed improved ferroelectric properties and lower leakage current density [9] whereas a combination of sputtered films of tetragonal PZT and PT composition has been reported to result in improved FOM for a pyroelectric application [10].

Ferroelectric and dielectric properties of alternating FE/AF layers were first reported by Dausch et al [6]. A slightly different structure where the FE layer is sandwiched between two AFE layers (all PZSTN) with improved fatigue properties due to antiferroelectric buffer between FE and electrodes having small stress during $180^{\circ}$ domain switching was reported by Jang et al [7]. In more recent study, Boldyreva et al [11] deposited thin films by sputtering with alternating FE/AF layers and showed the ferroelectric behaviour in PZ below certain film thickness or a phase coexistence of ferroelectric and antiferroelectric phase in PZ films [12].

In this study, the pyroelectric properties of the bi-layer films was investigated and compared with those of pure FE films.

\section{Experimental}

The antiferroelectric PZT 95/05 (AF) and ferroelectric PZT 30/70 films (FE) were deposited by chemical solution deposition (CSD) onto platinised silicon substrates. Details of sol preparation and thin film processing can be found in [13]. A typical single layer thickness of AF and FE layer was $50 \mathrm{~nm}$ and $70 \mathrm{~nm}$ respectively by a Dektak surface Profilometer and SEM crosssection images. In the bi-layer films, thickness of AF layers is $50 \mathrm{~nm}, 100 \mathrm{~nm}$ or $150 \mathrm{~nm}$ and FE 
layers $140,280,420,560$ and $700 \mathrm{~nm}$ on top of AF. In addition, pure FE films of the same thicknesses were deposited and served as a reference. For further comparison, three AF samples with 1 to 3 layers and $1 \mu \mathrm{m}$ thick were deposited.

The crystallographic structure and out-of-plane orientation of each film was determined by the standard $\theta-2 \theta$ X-ray diffraction (XRD) method on a Siemens D5005 diffractometer using $\mathrm{CuK} \alpha$ radiation and a Goebel mirror. For the measurement of electrical properties, a set of $\mathrm{Au} / \mathrm{Cr}$ electrode dots was evaporated onto the film surface through stainless steel shadow mask (diameter $0.75 \mathrm{~mm}$ ). The dielectric constant and loss tangent of the PZT films at $30 \mathrm{~Hz}$ were measured using a WK Wayne Kerr Precision Component Analyser 6425 after corona poled at $130^{\circ} \mathrm{C}$ and $22 \mathrm{kV}$ for $10 \mathrm{~min}$. The pyroelectric measurements were performed by varying the temperature of the sample by $\pm 2^{\circ} \mathrm{C}$ around mean temperature $25^{\circ} \mathrm{C}$ using the Byer-Roundy method [14]. This temperature range was chosen as pyroelectric properties near room temperature without the need of cooling the sample were of interest. The P-E loops were performed with the Radiant RT 66a test system. For the measurement of P-E loops at elevated temperatures the film was heated on a hotplate in the range between $20^{\circ} \mathrm{C}$ (room temperature) and $230^{\circ} \mathrm{C}$.

\section{Results and discussion}

The XRD diffractograms (not shown) of a bi-layer film revealed the peaks belonging to both AF and FE layers with their individual preferred orientations and no other phases. The FE and AF layers presented tetragonal and orthorhombic structure, respectively. The AF films showed a preferable growth in $(002)_{\mathrm{o}}$ direction while the FE films showed a mixed (111) $\mathrm{t}$ and $(100)_{\mathrm{t}}$ orientation. 
The P-E loops of bi-layer films with different AF layer thickness but same FE layer thickness $(140 \mathrm{~nm})$ are displayed in Fig. 1. The bi-layer films with 50 and $100 \mathrm{~nm}$ AF layer showed a typical ferroelectric hysteresis suggesting a dominant ferroelectric behaviour in these samples since the FE layers were much thicker than the AF layers. The bi-layer film with $50 \mathrm{~nm}$ AF layer showed typical features observed on FE P-E loop (not shown). The coercive field was however slightly higher than in the pure FE film probably due to the coupling of FE and AF layers and the contribution from the AF layer towards higher required critical coercive field. The polarization of the bi-layer film with $100 \mathrm{~nm}$ AF layer was lower than that of the bi-layer film with only $50 \mathrm{~nm}$ AF layer but the imprint showed tendency towards the negative coercive field. The P-E loop of the bi-layer film with $150 \mathrm{~nm}$ AF layer, thus with equal thicknesses of AF and FE layer, showed a narrowing of the loop at low field suggesting a double loop due to the AF layer. The coercive field of this film was similar to that of the bi-layer films with $100 \mathrm{~nm} \mathrm{AF}$ layer. As the thickness of either AF or FE layer in bi-layer films increased, the P-E loops showed more pronounced characteristics of that layer.

Pyroelectric coefficients for all bi-layer samples against the FE thickness are displayed in Fig. 2. The results of pure FE films are also included for comparison. The pyroelectric coefficient in pure FE films increases with thickness. The bi-layer films with 100 and $150 \mathrm{~nm} \mathrm{AF}$ layer showed minimum values at a certain FE thickness, around $400 \mathrm{~nm}$. The cause for this might be related to the high coercive field found for films and thus may result in insufficient poling of the films. Below or above this FE thickness the pyroelectric coefficient was found to increase. This suggests that the thickness ratio of the FE to AF layer plays an important role for properties of bi-layer films. The pyroelectric data for the bi-layer films with $50 \mathrm{~nm}$ AF layer with thinner FE layers could not be obtained due to the film being conductive. 
However, it must be noted that the orientations of the pure FE and FE in bi-layer films differ. In bi-layer films, FE layer was mainly $(100)_{\mathrm{t}}$ orientated while pure FE was (111)t. Comparing the pyroelectric coefficient of the bi-layer films with pure FE films showed enhanced pyroelectric properties of bi-layer films with $50 \mathrm{~nm}$ AF layer but for similar total thickness. The bi-layer films with 100 or $150 \mathrm{~nm} \mathrm{AF}$ layer, with the minimum at $400 \mathrm{~nm}$ FE layer, showed lower pyroelectric coefficient at this thickness but much higher pyroelectric coefficient below or above this particular FE thickness. To illustrate, the bi-layer film with 150 and $140 \mathrm{~nm} \mathrm{AF}$ and FE layer, respectively showed pyroelectric coefficient comparable to the pyroelectric coefficient of $1 \mu \mathrm{m}$ pure FE film for which a deposition of at least 10 layers is required. This is rather surprising considering that the polarization was found to be low for either type of thin films, the ferroelectric and antiferroelectric. Furthermore, the highest pyroelectric coefficient for pure PZT 30/70 films was $3.2 \times 10^{-4} \mathrm{Cm}^{-2} \mathrm{~K}^{-1}$ which lies between the values determined for bi-layer films with $150 \mathrm{~nm}$ or $100 \mathrm{~nm}$ thick AF layer and $900 \mathrm{~nm}$ thick FE layer but is lower than for bi-layer with $50 \mathrm{~nm}$ AF layer having same, $900 \mathrm{~nm}$ thick FE layer. Since the total thickness of bi-layer films is lower than for pure FE films and the pyroelectric coefficient is thickness dependent, probably higher values can be obtained for bi-layer films at the same thickness as pure FE films. All latter results suggest that the pyroelectric coefficient is a function of the thickness ratio of FE to AF layer.

Considering that the AF layer at the measurement temperature is not pyroelectric and should behave as a simple dielectric insulator, hence, it is surprising to detect any pyroelectric current at the measurement temperature. However, the antiferroelectric PZT 95/05 has a phase transition to ferroelectric phase above $110^{\circ} \mathrm{C}$ on heating and back to antiferroelectric phase at $60^{\circ} \mathrm{C}$ on cooling, according to the phase diagram [15]. The same phase transition from AF into FE can occur under sufficient electric field at room temperature but the film switches back into 
AF phase as soon as the field is removed. Since the films are poled under very high field and at $130^{\circ} \mathrm{C}$ before the pyroelectric measurement, the conditions for phase transition into a ferroelectric phase are given. However, there must be an additional mechanism that stabilizes the FE phase and prevents it from switching back into the AF phase after poling and being cooled down to room temperature.

To monitor the phase transitions of PZT 95/05 films, measurement of P-E loops on a $1 \mu \mathrm{m}$ AF film were performed in a temperature range. The partial results are displayed in Figure 3. Initially, a typical antiferroelectric double loop was observed at room temperature. More or less an unchanged loop was observed up to $110^{\circ} \mathrm{C}$ on heating. At and above $110^{\circ} \mathrm{C}$ a very narrow and distorted ferroelectric loop was observed (not shown). Above $220^{\circ} \mathrm{C}$ the ferroelectric phase transforms into paraelectric phase and a very open loop for a lossy dielectric was observed (not shown). Below $220^{\circ}$ (on cooling) the distorted ferroelectric loop was again detected and was observed in the whole temperature range down to room temperature. The partial switching back into the AF phase, of one branch of the loop (at negative field), was observed only the day after the measurement. The effect of phase transition into ferroelectric phase and the switching back into AF phase at lower temperatures than stated in the phase diagram for bulk PZT 95/05 was reported earlier by Handerek and Ujma [16] who found that the PZT 95/05 ceramic switched back into the $\mathrm{AF}$ phase only at $24^{\circ} \mathrm{C}$ on cooling. According to Handerek and Ujma this large diffuse phase transition is caused by the different local phase transition temperatures due to compositional fluctuations in the concentrations of $\mathrm{Pb}, \mathrm{Zr}$ and $\mathrm{Ti}$ ions, vacancies in $\mathrm{Pb}$ and $\mathrm{O}$ sublattices, local internal fields and mechanical stresses. Supposed the same phase transition temperature occurs in thin films the reason for not switching back into AF phase might be because this temperature is very close to the room temperature in the lab (usually around $22^{\circ} \mathrm{C}$ ). However, during the night the room temperature drops below this temperature so it is still 
surprising to find the film in the same FE phase the day after. Stabilising to FE phase at temperatures above $22^{\circ} \mathrm{C}$ can also explain why some pyroelectric current was measured on the same AF $1 \mu \mathrm{m}$ film. The signal of the pyroelectric response was very low but considering the low remanant polarization observed for that film after cooling its value is well in agreement. Similar AF-FE phase transition and stabilization of FE phase solid solutions for $\mathrm{Nb}$ and highly Sn doped PZT films were found by Xu et al [17] and were attributed to high tensile stress in thin films.

The same phase switching from AF to FE phase is likely to happen during poling of the bi-layer films but since the AF layer in bi-layer films is covered with FE layer it is more difficult to determine whether the film switched back into the AF phase. The XRD scan and careful peak deconvolution of the (100) peaks of the poled and unpoled film area for AF (50nm)/FE(420nm) film (Figure 4) revealed a strong $\mathrm{FE}(100)_{\mathrm{t}}$ peak and a smaller AF $(002)_{\mathrm{o}}$ peak before poling. After poling the integral intensity of $\mathrm{FE}(100)_{\mathrm{t}}$ peak was reduced by $85 \%$ and a new peak was observed at the position of $\mathrm{FE}(001)_{\mathrm{t}}$ peak having a three times higher intensity than the $\mathrm{AF}$ $(002)_{\mathrm{o}}$ peak before poling. The $\mathrm{AF}(002)_{\mathrm{o}}$ peak was not found after poling. Taking the domain switching of ferroelectric FE from $(100)_{\mathrm{t}}$ to $(001)_{\mathrm{t}}$ on poling into account the clear indication of phase switching from AF to FE requires the detection of a new (100) r peak after poling that belongs to the rhombohedral ferroelectric phase of PZT 95/05. However, the intensity of the $\operatorname{AFE}(002)_{\mathrm{o}}$ peak was quite low before poling so that the intensity of the new rhombohedral (100)r peak must be even lower and can be easily overshadowed by the background.

Both latter observations indicate that the absence of transition into the AF phase after the application of electric field above the phase transition temperature leads to switching of AF films into the FE phase which is stable at room temperature. It is not possible to say how long this effect lasted. Nevertheless, some pyroelectric measurements were repeated a few days after 
poling on the same samples and showed same results as only a few hours after poling. The XRD in Fig.4 was performed weeks after the bi-layer film was poled.

In contrast to the pure AF films the bi-layer films are sandwiched between the bottom electrode and the FE layer which may account for residual stress during poling that might stabilize the FE phase. Due to different thermal expansion coefficients and probably different residual stress between $\mathrm{AF}$ and $\mathrm{FE}$ layers there must be a component of in-plane strain during poling which might inhibit the switching back into the AF phase. Also, since the FE layer is polarized the ferroelectric domains at the interface FE/AF might induce a nonzero electric field in the AF layer which, if sufficient, might support switching into FE phase. Recently reported thickness dependent AF to FE in sputtered multilayer PZT AF-FE films was ascribed to strain between different layers [11]. In the latter work the film thickness was much smaller, below 10 $\mathrm{nm}$, and the stack of alternating PZ and rhombohedral PZT was used. PZ films by Boldyreva et al were prepared by a different deposition method and they were not exposed to electric field and temperature which can explain why in their work the FE behaviour was only observed in films thinner than $10 \mathrm{~nm}$ while in this work PZT 95/05 films as thick as 1 micron show FE behaviour.

Accordingly, it is possible that the in-plane strain in bi-layer films changed after poling which can account for the dependence of the pyroelectric coefficient on the bi-layer thickness ratio. The stress measurement of AF thin films before poling showed an increase of residual stress with increasing thickness for 100 to $200 \mathrm{~nm}$ thin films [18], whereas in the FE films a tendency to high residual stress within the first layer was observed which decreases upon coating of more layers [19]. Thus the residual stress in the bi-layer films is probably rather complicated and the phase transition of the AF layer during poling might have consequences for the stress state in the FE layer. 
Although the remanant polarization of switched FE phase of the AF layer is not high (around $10 \mu \mathrm{C} / \mathrm{cm}^{2}$ ), together with the highly (001) orientated FE layer after poling it can account for the enhanced pyroelectric coefficient of bi-layer films. Thus, such double contribution of the FE orientation and switching of AF layer can explain why it shows the lowest pyroelectric coefficient at certain thickness but enhancement above or below such thickness. The pyroelectric coefficient of PZT 30/70 in this work is in agreement with the results for PZT 30/70 $700 \mathrm{~nm}$ thick films which was $2.11 \times 10^{-4} \mathrm{Cm}^{-2} \mathrm{~K}^{-1}[13]$.

There is always a concern about this pyroelectric enhancement, which probably is due to the existence of defect charge or pinning charge in the interface between AF and FE layers. However, if the defect charge caused the enhancement of pyroelectric coefficient of FE films, a linear increase of pyroelectric coefficient with FE layer thickness should have been observed because the defect charge should be the same for films with same AF thickness. However, in our case a reduction of pyroelectric coefficient was observed before it increased again at greater thickness.

Table I lists the data of dielectric properties for poled bi-layer films and also poled pure PZT 30/70 films for comparison. Unpoled data were not listed. The dielectric constant and loss at $30 \mathrm{~Hz}$ was varying with $\mathrm{FE} / \mathrm{AF}$ thickness ratio for all bi-layer films. The dielectric constant in bi-layer films with 150, 100 and $50 \mathrm{~nm}$ AF layers decreases accordingly. Not much difference in the dielectric constant before and after poling was observed for films with 100 and $150 \mathrm{~nm} \mathrm{AF}$ layer whereas much lower dielectric constant was measured after poling for films with 50nm AF layer. The $\tan \delta$ graphs for bi-layer films show similar trends as the dielectric constant and vary with thickness. At lower FE thicknesses the tan $\delta$ is higher for bi-layer films than for pure FE films but it decreases and approaches the values for pure FE films at higher FE thicknesses. 
The dielectric constant for all bi-layer films was lower than that of the pure FE films, as expected since the dielectric constant of AF films is lower, around 200, except for two values of bi-layer films with $150 \mathrm{~nm}$ AF layer. The increase of dielectric constant of pure FE films with thickness can be ascribed to residual stress as was already mentioned for polarization increase with increasing thickness. The variation however is probably due to different orientation between individual films which can account for different intrinsic values of the dielectric constant. It is also noteworthy that the determined dielectric constant for FE films is rather high since values around 400 up to 600 are usually reported for $1 \mu \mathrm{m}$ thick PZT 30/70 films [13, 20].

The decrease of the dielectric constant after poling in bi-layer films and FE films can be due to both intrinsic and extrinsic contributions. The intrinsic one is due to the orientation change from (100) into (001), orientation which lowers the dielectric constant due to lower values for corientated (198) than for a-orientated (499) tetragonal PZT films [21]. The extrinsic contribution is the removal of $180^{\circ}$ domain walls during poling, which results in lower dielectric constant.

To investigate the contribution of the AF layer, the dielectric constant of bi-layers was modelled according to the equivalent circuit of linear series capacitors based on pure AF and FE films as following:

$$
\varepsilon=\frac{\varepsilon_{A F} \varepsilon_{F E} t}{\varepsilon_{A F} t_{A F}+\varepsilon_{F E} t_{F E}}
$$

with indices AF and FE denoting the dielectric constant and thickness of the corresponding layer, $t$ the total bi-layer thickness and $\varepsilon$ the dielectric constant of the bi-layer. However, the modelled and experimental results of dielectric constant for bi-layer films do not agree, indicating that the model we were using was too simple to reflect the true case. 
The figure of merit $F_{D}(\mathrm{FOM})$ was calculated for all samples at $30 \mathrm{~Hz}$ according to [22]:

$$
F_{D}=\frac{p^{\prime}}{c^{\prime}\left(\varepsilon \varepsilon_{0} \tan \delta\right)^{0.5}}
$$

where $p^{\prime}$ denotes the pyroelectric coefficient and $c^{\prime}$ is the specific heat which is $2.6 \times 10^{6} \mathrm{~J} / \mathrm{m}^{3} \mathrm{~K}$ for PZT 30/70 [13]. The highest FOM at $30 \mathrm{~Hz}$ was $2 \times 10^{-5} \mathrm{~Pa}^{-0.5}$ for $\mathrm{AF}(100) / \mathrm{FE}(900)$ bi-layer film which is nearly double the value, for pure $\mathrm{FE}(820 \mathrm{~nm})$ film or still higher than $1.5 \times 10^{-5} \mathrm{~Pa}^{-}$ 0.5 determined for pure FE $1150 \mathrm{~nm}$ thick film. However, these figures are lower than the values determined for Mn doped PZT film which was $3.52 \times 10^{-5} \mathrm{~Pa}^{-0.5}$ at $30 \mathrm{~Hz}$ however still higher than the $F_{D}$ of pure FE films. The reason for lower $F_{D}$ in this work compared to work of Zhang et al [13] for Mn doped PZT, although similar highest pyroelectric coefficients were determined, is due to the higher dielectric constant of the FE films in this work. Also, the dielectric constant and loss are much smaller for PMZT which lead to significant increase of the figure of merit of PMZT films.

\section{Conclusions}

Bi-layer films of $\mathrm{AF} / \mathrm{FE}$ were prepared and for the purpose of comparison, pure $\mathrm{AF}$ and $\mathrm{FE}$ films were also prepared. Their microstructure and electrical properties were investigated. It was found that the ferroelectric, dielectric and pyroelectric properties of FE films were dependent on film thickness due to the decrease of residual stress with thickness.

Enhanced pyroelectric coefficient was found for the majority of bi-layer films when compared to pure FE films of similar thickness. However, it was not possible to model the bilayer films as two linear series capacitors. The switching of the AF phase to FE phase and only partial switching back into AF phase were observed for $1 \mu \mathrm{m}$ pure AF film. The evidence of 
pyroelectric current at temperatures close to the room temperature indicates that the AF layer in bi-layer films is not behaving as an insulator, which is rather unexpected from AF materials, but must have transformed into FE phase. The stabilization of the FE phase can have several causes such as residual strain, defects mobility or the electric polarization influence from the FE layer. $F_{D}$ was higher for most of the bi-layer films in comparison to pure, similar thick FE films. The introduction of antiferroelectric layer into ferroelectric film structure seems a promising method for enhancement of the pyroelectric coefficient of thin films. Furthermore, manufacturing a bilayer of AF and PMNT films is an appealing option to even further enhance the pyroelectric coefficient and FOM and is the subject of future work.

\section{Acknowledgements}

The authors thank and acknowledge Dr. A. Jiang for fruitful discussion and EPSRC grants (EP/EO35043/1) for financial support.

\section{References}

[1] S.S. Sengupta, D. Roberts, J.-F. Li, M.C. Kim, D.A. Payne, J. Appl. Phys. 78, 1171 (1995).

[2] J.F. Li, D.D. Viehland, T.Tani, C.D.E. Lakeman, D.A. Payne, J. Appl. Phys. 75, 442 (1994).

[3] B. Xu, L. Eric Cross, J. J. Bernstein, Thin Solid Films, 377-378, 712 (2000).

[4] K. Yamakawa, S. Trolier-McKinstry, and J. P. Dougherty, Proceedings of the Tenth IEEE ISAF, Vol. 1, 405 (1996).

[5] A. S. Mischenko, Q. Zhang, J. F. Scott, R. W. Whatmore, N. D. Mathur, Science, 311, 1270 (2006). 
[6] D.E. Dausch, F. Wang, G.H. Haertling, IEEE International Symposium on Applications of Ferroelectrics, 701 (1994).

[7] J.H. Jang, K.H. Yoon, Thin Solid Films 401, 67 (2001).

[8] K. Boldyreva, D. Bao, G. Le Rhun, L. Pintilie, M. Alexe, D. Hesse, J. Appl. Phys. 102, 044111 (2007).

[9] F. C. Kartawidjaja, C. H. Sim, and J. Wang, J. Appl. Phys. 102, 124102 (2007).

[10] W. Liu, J.-S. Ko, W. Zhu, Thin Solid Films 371, 254 (2000).

[11] K. Boldyreva, L. Pnitilie, A. Lotnyk, I.B. Misrlioglu, M. Alexe, D. Hesse, Appl. Phys. Lett. 91, 122915 (2007).

[12] L. Pintilie, K. Boldyreva, M. Alexe, D. Hesse, J. Appl. Phys. 103, 024101 (2008).

[13] Q. Zhang and R.W. Whatmore, J. Appl. Phys. 94(8), 5228 (2003).

[14] R. L. Byer, C.B. Roundy, IEEE Trans. Sonics Ultrason. 19, 333 (1972).

[15] P. Capper, S.O. Kasap, Springer Handbook of Electronic and Photonic Materials, Springer, New York, 597 (2006).

[16] J. Handerek, Z. Ujma, J. Phys.: Condens. Matter 7, 1721 (1995).

[17] B. Xu, Y. Ye, L.E. Cross, J. Appl. Phys. 87, 2507 (2000).

[18] S. Corkovic, Q. Zhang, Functional Materials Letters, Vol. 1, No. 1, 13 (2008).

[19] S. Corkovic, R.W. Whatmore, Q. Zhang, J. Appl. Phys. 103, 084101 (2008).

[20] J.-S. Yang, S.-H. Kim, D.-Y. Park, E. Yoon, J.-S. Park, T.-S. Kim, S.-G. Kang, J. Ha, Jpn. J. Appl. Phys. 42, 5956 (2003).

[21] X.H. Du, U, Belegundu, K. Uchino, J. Appl. Phys. 36, 5580 (1997).

[22] R.W. Whatmore, Rep. Prog. Phys. 49, 1335 (1986). 
Table I: Dielectric properties of bi-layer films and pure PZT 30/70 films at $30 \mathrm{~Hz}$.

\begin{tabular}{|c|c|c|c|}
\hline & \multicolumn{3}{|c|}{$\begin{array}{l}\text { Dielectric constant (loss tan } \delta \text { ) of bi-layer } \\
\text { films }\end{array}$} \\
\hline $\begin{array}{l}\text { FE thickness } \\
\text { thickness } \\
{[\mathrm{nm}]}\end{array}$ & 50 & 100 & 150 \\
\hline 280 & $236(0.028)$ & $228(0.008)$ & $443(0.009)$ \\
\hline 420 & $292(0.014)$ & $363(0.015)$ & $405(0.013)$ \\
\hline 560 & $404(0.012)$ & $330(0.008)$ & $660(0.008)$ \\
\hline 920 & $317(0.016)$ & $364(0.011)$ & $462(0.010)$ \\
\hline Thickness [nm] & \multicolumn{3}{|c|}{$\begin{array}{l}\text { Dielectric constant (loss tan } \delta \text { ) of pure PZT } \\
30 / 70 \text { films }\end{array}$} \\
\hline 380 & \multicolumn{3}{|c|}{$445(0.014)$} \\
\hline 580 & \multicolumn{3}{|c|}{$530(0.012)$} \\
\hline 820 & \multicolumn{3}{|c|}{$515(0.014)$} \\
\hline 1150 & \multicolumn{3}{|c|}{$645(0.011)$} \\
\hline
\end{tabular}

\section{Figure Captions:}

Figure 1: P-E loops of bilayer films with a) different AFE layer thickness and same FE layer thickness $(140 \mathrm{~nm})$.

Figure 2: Pyroelectric coefficient of bi-layer films vs the FE layer thickness. The pyroelectric coefficient of pure FE films is included for comparison.

Figure 3: P-E loops of $1 \mu \mathrm{m}$ AF film measured at room temperature before and after it was heated to $220^{\circ} \mathrm{C}$ and cooled to room temperature. 
Figure 4: Diffractorgrams of bi-layer film with $50 \mathrm{~nm}$ AF layer and $420 \mathrm{~nm}$ FE layer before and after poling. The diffractorgrams of pure AF and FE films are included for comparison. Please note that for the bi-layer film the collimator size was much smaller compared to pure films and hence result in different intensity. 